\title{
Immunocytochemistry in the diagnosis of Whipple's disease
}

\author{
DAVID J EVANS, ${ }^{*}$ MUFEED H ALI $\dagger$ \\ From the *Department of Histopathology, Hammersmith Hospital, London W12, and †Wexham Park \\ Hospital, Slough, Berkshire
}

SUMMARY In six cases of Whipple's disease the characteristic macrophages could be stained immunocytochemically using a first layer antiserum to group B streptococci. Other types of periodic acid Schiff positive macrophages were negative. The staining appears to be due to a rhamnose containing polysaccharide as shown by a blocking test. The method may be useful for confirming the diagnosis in rectal biopsy and peripheral nodes, especially if small intestinal findings are atypical.

The histopathological diagnosis of Whipple's disease is usually straightforward when the submitted material consists of a small intestinal biopsy or a mesenteric lymph node. In both instances there is pronounced distortion of tissue architecture; large lipid containing spaces can be seen; and the characteristic macrophages are present, the particles in their cytoplasm staining brilliantly with the diastase periodic acid Schiff (DPAS) method.'

Although histiocytes typical of the disease are present in other tissues such as peripheral lymph nodes and rectal biopsies, they are easily overlooked (Fig. 1) or confused with histiocytes containing other DPAS positive material such as prolipofuscin, lipofuscin, or mucin. Such confusion can be resolved by the use of conventional stains, ${ }^{2}$ though none of these gives a preferentially positive result with the macrophages of Whipple's disease.

It has been noted, however, that the macrophages react with antisera which are specific to group $B$ or group G streptococci. ${ }^{3}$ We have tested a group B specific streptocuccal antiserum on a further series of cases of Whipple's disease and on other types of DPAS positive macrophages and have investigated the antigenic specificity of the reaction.

\section{Material and methods}

The material was obtained from the files of the Hammersmith Hospital and London Hospital pathology departments in the form of paraffin embedded blocks; the tissue had been primarily

Accepted for publication 4 December 1984

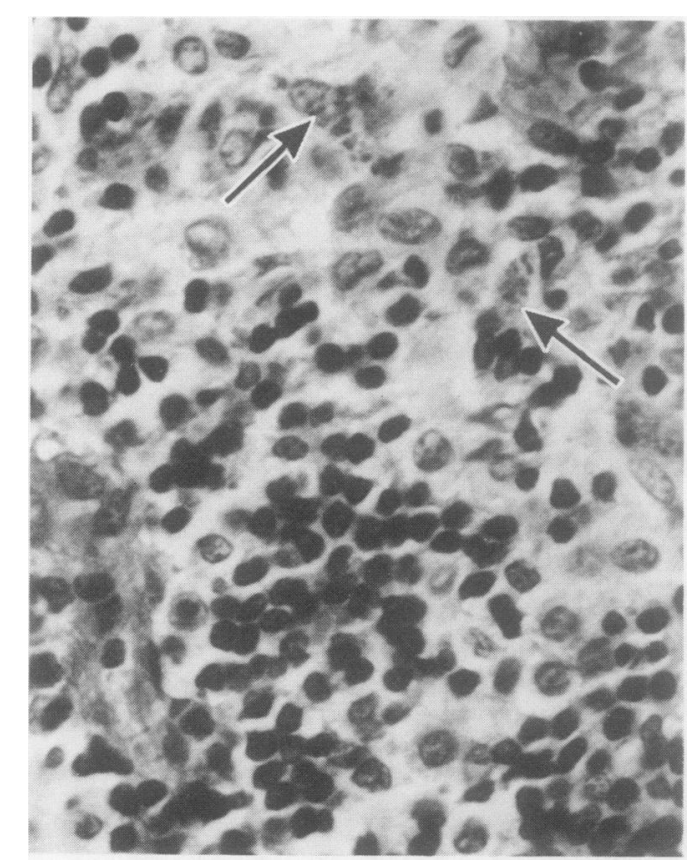

Fig. 1 Peripheral lymph node from a case of Whipple's disease. Scattered macrophages contain the diastase-periodic acid Schiff (DPAS) positive granules (two are arrowed). DPAS. $\times 600$.

fixed either in formalin or in formol-mercury. The tissue consisted of the following: (a) four rectal biopsies from different cases of Whipple's disease; (b) two small intestinal biopsies from different cases of Whipple's disease; $(c)$ one small intestinal biopsy 
and one peripheral lymph node from a case of Whipple's disease; $(d)$ one necropsy case of Whipple's disease with lymph node, large intestine, small intestine, and liver available; $(e) 10$ rectal biopsies containing muciphages as shown by mucicarmine; (f) 10 rectal biopsies containing lipofuscin, as shown by autofluorescence; $(g)$ one bladder showing malakoplakia.

Four micron thick paraffin sections were cut and passed through xylene and graded alcohols to phosphate buffered saline (PBS). Mercury was removed where necessary with iodine and sodium thiosulphate.

Staining for fluorescence microscopy was performed by conventional methods ${ }^{4}$ using a rabbit antiserum to group B streptococci (Wellcome Research Laboratories), diluted 1/20 in PBS, as first layer and a fluorescein labelled antirabbit immunoglobulin antiserum (North East Biomedical Laboratory), diluted 1/100 in PBS, as second layer.

For immunoperoxidase staining a peroxidaseantiperoxidase technique was used, ${ }^{s}$ with the first layer being the rabbit antiserum at a dilution of $1 / 500$. The second layer was a swine antirabbit antiserum (1/200) and the third layer peroxidase-rabbit antiperoxidase complexes $(1 / 300)$, both from Dakopatts. Endogenous peroxidase was blocked by preincubation with $0.3 \%$ hydrogen peroxide in PBS. In both systems normal rabbit serum was used as a negative control.

To confirm the carbohydrate specificity of the first layer rabbit antibody, sections from the necropsy case of Whipple's disease which reacted positively with the antibody were incubated with it in the presence of $0.1,0.2$, and $0.5 \mathrm{M}$ rhamnose (6deoxymannose), glucose or mannose in PBS. They were then washed with PBS and incubated with the fluorescein labelled goat antirabbit immunoglobulin antiserum and processed as before.

\section{Results}

Tissues containing muciphages, lipofuscin containing macrophages, or showing malakoplakia were uniformly negative.

In all the cases of Whipple's disease examined, there was unequivocally positive staining of the characteristic macrophages using the antiserum to group B streptococci (Fig. 2). Some macrophages with positive staining were identified in all the tissues listed above. The staining appeared for the most part to be confined to histiocytes, though in the small intestine occasional positivity was observed within the epithelium (Fig. 3). It was not possible to recognise with certainty extracellular material. In histiocytes the staining was not always strong and

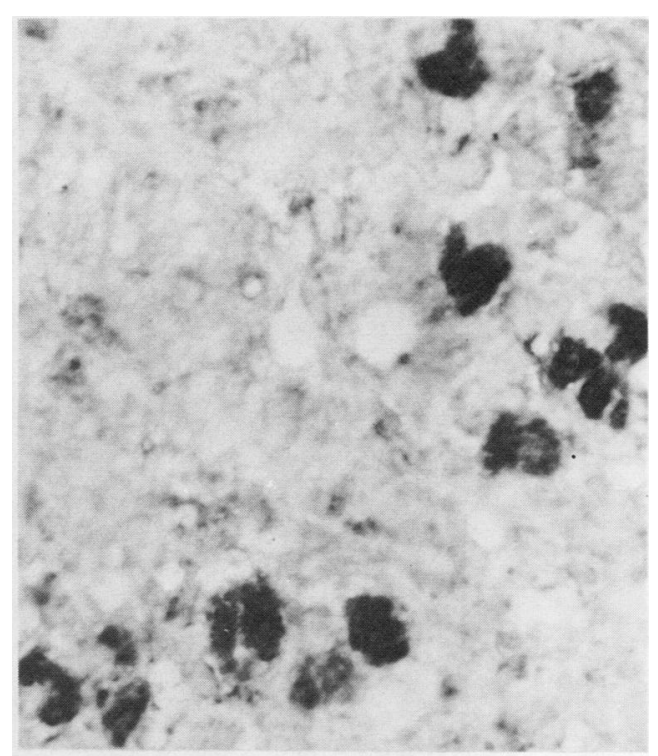

Fig. 2 Section of same node as in Fig. 1 stained with rabbit anti-group $B$ streptococcus antiserum and $a$ peroxidase-antiperoxidase (PAP) technique, without nuclear counterstain. Many of the macrophages show specific staining, though of varied intensity. PAP technique. $\times 600$.

was not uniform within these cells.

Staining of histiocytes was totally blocked by $0.5 \mathrm{M}$ rhamnose but not by other carbohydrate solutions.

\section{Discussion}

Despite much work, the nature of the organism or organisms causing Whipple's disease is unclear. The present investigation confirms that an antiserum specific to group B streptococci stains the macrophages of Whipple's disease in a variety of organs, and that similar staining is not seen with other DPAS positive macrophages even in malakoplakia, in which the intracellular material is known to be derived from bacteria. ${ }^{6}$ This type of antiserum recognises polysaccharides containing rhamnose subunits, ${ }^{7}$ and the capacity of solutions of rhamnose, but not of two other monosaccharide solutions in similar concentration, to block the staining of the Whipple's disease macrophages indicates that they contain such a polysaccharide. The necessity for high concentrations of the monosaccharide in solution may reflect the low affinity of the antibody for the monosaccharide as compared with polysaccharide.

The universal presence of rhamnose in Whipple's disease seems to favour the view that a single organ- 


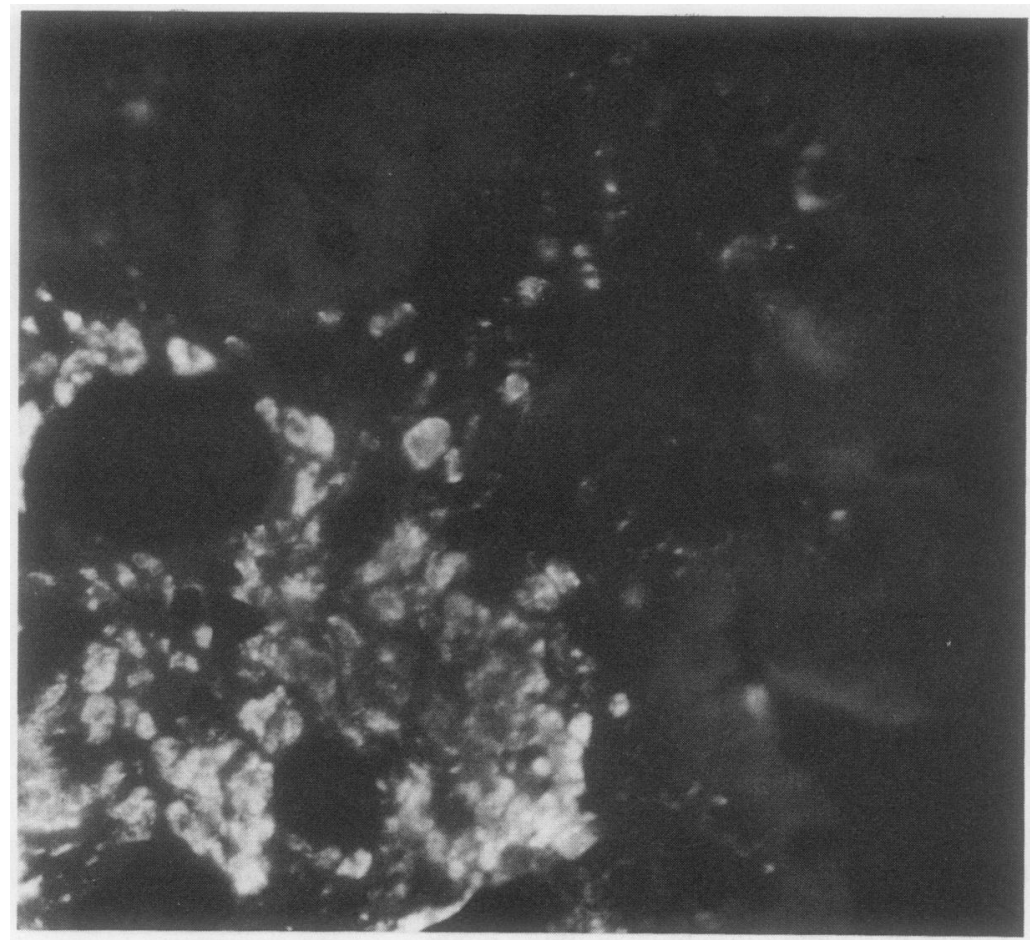

Fig. 3 Small intestinal biopsy stained by indirect immunoftuorescence with rabbit anti-group B streptococcus antiserum. Most of the positivity is within macrophages, though some intraepithelial staining is present. Immunofuorescence. $\times 150$.

ism is responsible for the disease. Although this sugar does not occur normally in mammalian tissue, it is found in many bacterial polysaccharides. As well as the group B and G streptococci, other streptococci, Shigella flexneri, some lactobacilli, and Corynebacteriaceae contain rhamnose, and therefore its presence does not identify a particular organism. The material in the macrophages is undergoing lysosomal digestion, and the irregularity of staining with the antiserum may be due to sequential changes in the bacterial carbohydrates during this digestion.

Antisera to group B streptococci appear to provide a reliable positive stain for the macrophages of Whipple's disease and may prove useful for positive identification of the disease on material such as lymph nodes, rectal biopsies, or brain, especially if the classic cells are sparse or absent in small intestine.

We thank Dr D Pollock for the generous gift of material, Mr W Hinkes for the photography, Miss S van Noorden for expert technical help, and Mrs P
Weller for secretarial assistance.

\section{References}

' Enzinger FM, Helwig EB. Whipple's disease: a review of the literature and report of fifteen patients. Virchows Arch [Pathol Anat] 1963;336:236-69.

${ }^{2}$ Azzopardi JG, Evans DJ. Mucoprotein containing histiocytes (muciphages) in the rectum. J Clin Pathol 1966; 19:368-74.

${ }^{3}$ Kent SP, Kirkpatrick M. Whipple's disease: Immunological and histochemical study of 8 cases. Arch Pathol Lab Med 1980; 104:544-7.

+ Polak JM, Van Noorden S. Immunocytochemistry. Bristol: Wright, 1983:34.

' Comer GM, Brandt LJ, Abissi CJ. Whipple's disease: a review. J Gastroenterol 1983; 78:107-14.

- Lou TY, Teplitz C. Malakoplakia. Pathogenesis and ultrastructural morphogenesis: a problem of altered macrophage (phagolysosomal) response. Hum Pathol 1974;5:191-207.

' Curtis SN, Krause RM. Antigenic relationships between groups B and G streptococci. J Exp Med 1964;120:629.

Requests for reprints to: Professor DJ Evans, Department of Histopathology, Royal Postgraduate Medical School, Hammersmith Hospital, Ducane Road, London W12 0HS, England. 